\title{
A generic method for the enumeration of various classes of directed polycubes
}

\author{
Jean-Marc Champarnaud|j Jean-Philippe Dubernard| Hadrien Jeanne $\beta^{\beta}$
}

LITIS, Université de Rouen, Faculté des Sciences et Techniques

received $5^{\text {th }}$ June 2011, revised 27th January 2013, accepted $2^{\text {nd }}$ March 2013.

Following the track of polyominoes, in particular the column-by-column construction of Temperley and its interpretation in terms of functional equations due to Bousquet-Mélou, we introduce a generic method for the enumeration of classes of directed polycubes the strata of which satisfy some property $\mathcal{P}$. This method is applied to the enumeration of two new families of polycubes, the s-directed polycubes and the vertically-convex s-directed polycubes, with respect to width and volume. The case of non-directed polycubes is also studied and it is shown that the generic method can be applied in this case too. Finally the general case of $d$-dimensional polycubes, with $d \geq 4$, is investigated, and the generic method is extended in order to handle the enumeration of classes of directed $d$-polycubes.

Keywords: Directed polycubes, enumeration, generating function, dimension 3, dimension $d$

\section{Introduction}

In the Cartesian plane $\mathbb{N}^{2}$, a polyomino is a finite connected union of elementary cells (unit squares) without cut point and defined up to a translation. Even if they have been studied for a long time in combinatorics, no exact formula is known for counting general polyominoes but many results have been found concerning certain classes of polyominoes, see for instance [BM96] or [Fer04]. There also exist several classical tools to enumerate polyominoes. Among them, let us cite, for instance, the Temperley methodology [Tem56], the DSV methodology [DV84], the $q$-grammars [DF93], or the methodology of Bousquet-Mélou [BM96].

Polyominoes also have a 3-dimensional equivalent: the 3-dimensional polycubes (or polycubes for short) [Lun71] (see Figure 1]. If we consider, now, that an elementary cell is a unit cube, then a polycube is a face-connected finite set of elementary cells defined up to a translation in $\mathbb{N}^{3}$. As polyominoes, polycubes appear in statistical physics, more precisely in the phenomenon of percolation (see [BH57] for example). A lot of studies have led to count polycubes with respect to their number, $n$ say, of cells. The first values were found in 1972 up to $n=6$ [Lun71] and the last one (to our knowledge) in 2006, up to $n=18$ [AB09].

\footnotetext{
†jean-marc.champarnaudeuniv-rouen.fr

$\ddagger$ jean-philippe.dubernard@univ-rouen. fr

$\S$ hadrien. jeanneduniv-rouen.fr
} 
The notion of polycube can be extended to dimension $d$, with $d \geq 3$; $d$-dimensional polycubes (or $d$ polycubes for short) are used in an efficient model of real-time validation [LG08], as well as in the representation of finite geometrical languages [CDJ09, Jea10].

A particular class of polycubes, the plane partitions, has been studied for a long time (see [CLP98, Bre99. Sta01] for instance). Very recently, we have studied two new classes of polycubes: the plateau polycubes, that appear in a particular model of real-time validation, have been enumerated with respect to volume and width whereas there is only an asymptotic result for the parallelogram polycubes [CDJ10]. No other subclass of polycubes seems to have been examined.

In the next section, we define extensions of classes of directed polyominoes to the 3-dimensional case. Then, in Section 3, we present a new generic method to enumerate various classes of directed polycubes the strata of which satisfy a given property. In Section 4 this method is applied to enumerate two classes of directed polycubes: the s-directed polycubes and the vertically-convex s-directed polycubes. We show in Section 5 that it can also be applied to certain families of non-directed polycubes, such as the verticallyconvex pseudo-directed polycubes. Finally, Section 6 is devoted to the general case of $d$-dimensional polycubes, $d \geq 3$; the generic method is applied to a family of $d$-dimensional polycubes that generalize the 3-dimensional s-directed ones.

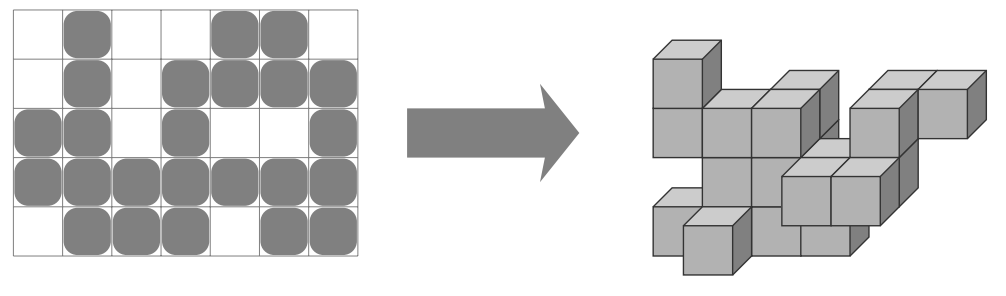

Figure 1: An ordinary polycube.

\section{Preliminaries}

A polyomino is said to be row-convex (resp. column-convex) if its intersection with any horizontal (resp. vertical) strip is convex. It is said to be directed if each of its cells can be reached from a distinguished cell, called root, by a path only made of East and North steps.

Let $(0, \vec{i}, \vec{j}, \vec{k})$ be an orthonormal coordinate system where $\vec{i}$ and $\vec{k}$ are horizontal vectors and $\vec{j}$ the only vertical vector. As for polyominoes, several parameters can be defined for a polycube. The volume is the number of elementary cells, the width (resp. height, depth) is the difference between the greatest and the smallest indices of the polycube according to $\vec{i}$ (resp. $\vec{j}, \vec{k}$ ). A polycube is said to be horizontally-convex if its intersection with any horizontal plane $(\vec{i}, \vec{k})$ is a row-convex polyomino; it is said to be verticallyconvex if its intersection with any vertical plane $(\vec{i}, \vec{j})$ is a column-convex polyomino. An example of horizontally-convex polycube is given in Figure 2. Similarly, a polycube is said to be antihorizontallyconvex if its intersection with any horizontal plane $(\vec{i}, \vec{k})$ is a column-convex polyomino; it is said to be antivertically-convex if its intersection with any vertical plane $(\vec{j}, \vec{k})$ is a column-convex polyomino.

Definition 2.1 A polycube is said to be convex if it is both horizontally, antihorizontally, vertically and antivertically convex. 

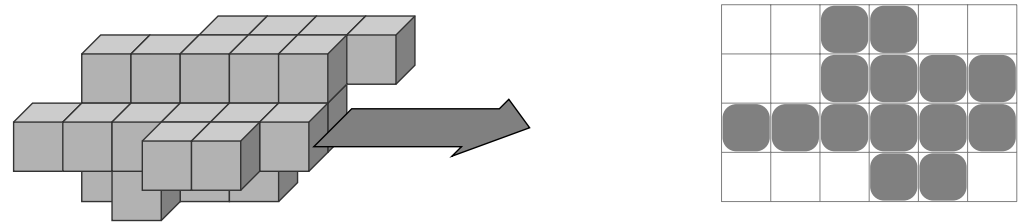

Figure 2: A horizontally-convex polycube.

The 2-dimensional notion of step is extended as follows. An East (resp. North, Ahead) step is a movement of one unit in $\vec{i}$-direction (resp. $\vec{j}$-direction, $\vec{k}$-direction). So, a polycube is said to be directed if each of its cells can be reached from a distinguished cell, called root, by a path only made of East, North and Ahead steps.

The 2-dimensional notion of column is extended as follows. A stratum is a polycube of width 1 . Hence a stratum can be directed or horizontally (resp. vertically, antihorizontally, antivertically) convex. Two examples of strata are given in Figure 3 . The notion of stratum allows us to define two new families of polycubes.

Definition 2.2 A polycube is said to be s-directed if the two following conditions are satisfied:

(1) its strata are directed,

(2) its strata are glued together so that the polycube is directed.

Definition 2.3 A polycube is said to be vertically convex s-directed if the two following conditions are satisfied:

(1) its strata are vertically convex directed,

(2) its strata are glued together so that the polycube is directed.

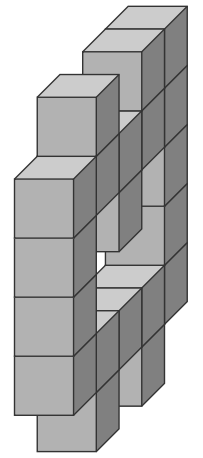

(1)

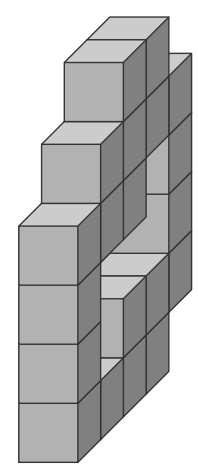

(2)

Figure 3: An ordinary stratum (1) and a directed stratum (2). 


\section{A generic method to enumerate families of directed polycubes}

The method that is presented here to enumerate families of directed polycubes is based on the columnby-column decomposition of polyominoes, known as "Temperley methodology", and on its interpretation in terms of functional equations due to Bousquet-Mélou [BM96]. These two techniques are extended to a slice-by-slice construction of polycubes. After a recall of notation and definitions, we describe how to decompose a directed polycube into its strata; we then show how to translate this decomposition into a functional equation and how to solve it.

\subsection{Definitions and notation}

Let $\mathbb{R}[[s, t, p, q]]$ be the algebra of formal power series in the variables $s, t, p$ and $q$, with real coefficients. Let $X(s, t, p, q)$ be such a series, then we use $\frac{\partial X}{\partial q}(s, t, p, q)$ to denote its derivative with respect to $q$. If $E$ is an expression depending on $q$, the value of $E$ at $q=i$ is denoted by $\left.E\right|_{q=i}$. Let $\mathcal{A}$ be the sub-algebra of the series $S$ of $\mathbb{R}[[s, t, p, q]]$.

The height of a stratum $S$ is denoted by $h(S)$, its volume by $v(S)$ and the area of its rightmost face by $a(S)$. These notations can be extended to polycubes. Moreover, the width of a polycube $P$, i.e. the number of strata is denoted by $l(P)$.

The generating series of a set $\mathcal{E}$ of directed polycubes is the following formal power series, which lies in $\mathcal{A}$ :

$$
\sum_{P \in \mathcal{E}} s^{h(p)} t^{l(P)} p^{v(P)} q^{a(P)} .
$$

\subsection{The generic method}

Given a property $\mathcal{P}$, let $\mathcal{C}_{\mathcal{P}}$ be the class of directed polycubes the strata of which satisfy the property $\mathcal{P}$. The generic method allows us to enumerate the class $\mathcal{C}_{\mathcal{P}}$.

The first step consists in enumerating directed strata satisfying the property $\mathcal{P}$. Let $Q(p, q)$ be the generating function of this family of directed strata with respect to volume and area of the rightmost face. Sincea stratum is obtained by substituting each unit square of a polyomino by a unit cube, if it statisfies a property $\mathcal{P}$ in dimension 3 , the corresponding polyomino also statisfies the property $\mathcal{P}$ in dimension 2 (see Fig. 4).

To begin with, we have to provide an expression for the generating function $P(q)$ of directed polyominoes satisfying the property $\mathcal{P}$ with respect to area. The expression $Q(p, q)$ follows from $P(q)$ by noting that the area of a polyomino is equal to the volume of the corresponding stratum. Note that the area of the rightmost face of the stratum is also equal to the area of the corresponding polyomino, as can be seen in Figure 4.

Finally, an expression for $Q(p, q)$ is given by:

$$
Q(p, q)=P(p q)
$$

Let us now focus on the enumeration of directed polycubes the strata of which satisfy the property $\mathcal{P}$. Let $R(t, p)$ be the generating function of the class $\mathcal{C}_{\mathcal{P}}$, with respect to width and volume. Given a polycube $C \in \mathcal{C}_{\mathcal{P}}$, two cases have to be distinguished:

- The polycube $C$ is reduced to a stratum: this case is enumerated by $t Q(p, 1)$. 

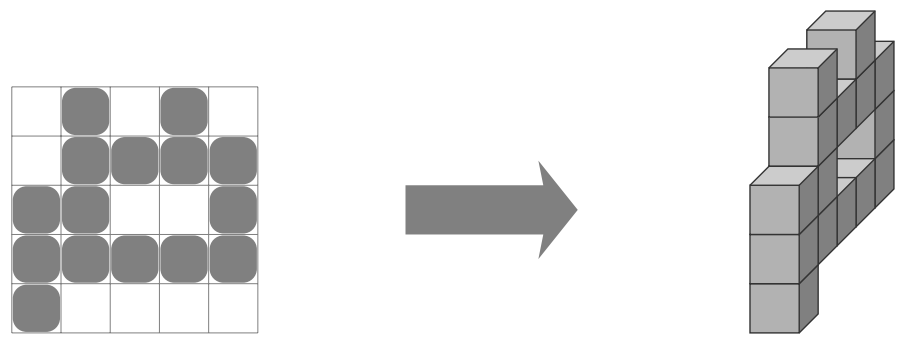

Figure 4: From a polycube to a stratum.

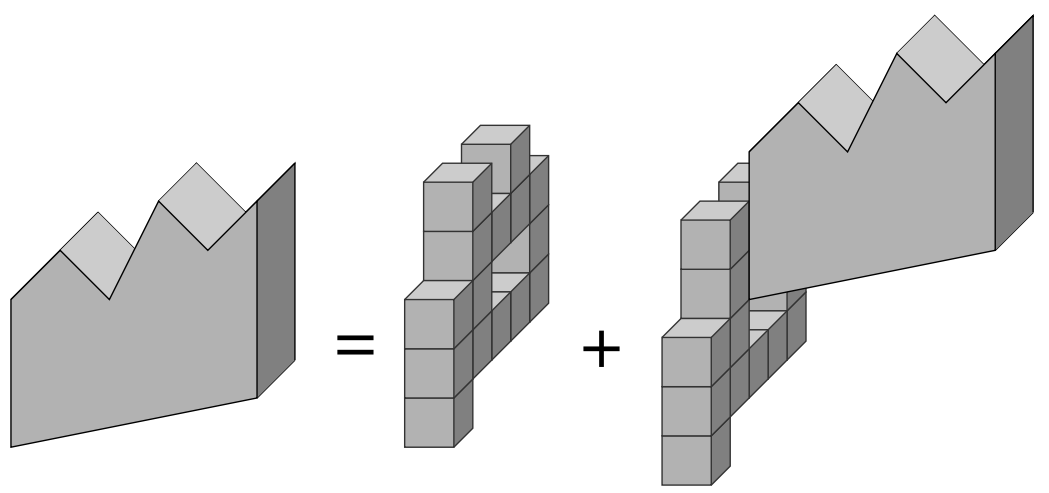

Figure 5: Decomposition of a polycube $C \in \mathcal{C}_{\mathcal{P}}$.

- The polycube $C$ can be decomposed into a stratum and a polycube of $\mathcal{C}_{\mathcal{P}}$ the root cell of which is glued on the stratum: this construction is explained in Figure 5

As a consequence, the number of possible stickings is equal to the number of unitary faces composing the area of the rightmost face of the stratum. This case is therefore enumerated by

$$
\left.t \frac{\partial Q}{\partial q}(p, q) R(t, p)\right|_{q=1} .
$$

The factor $t$ comes from the fact that we add a stratum; the term $\frac{\partial Q}{\partial q}(p, q)$ represents the number of possibilities to glue the polycube on the rightmost face of the stratum and the factor $R(t, p)$ is due to the gluing of the polycube.

We finally obtain a functional equation satisfied by the generating function of the class $\mathcal{C}_{\mathcal{P}}$.

Proposition 3.1 The generating function $R(t, p)$ of directed polycubes the strata of which satisfy the property $\mathcal{P}$, with respect to width and volume, admits the following functional equation:

$$
R(t, p)=t Q(p, 1)+\left.t\left(\frac{\partial Q}{\partial q}(p, q) R(t, p)\right)\right|_{q=1} .
$$


It is possible to solve this equation according to the generating function $P(q)$ of directed polyominoes satisfying the property $\mathcal{P}$. As a consequence, we obtain the following theorem:

Theorem 3.1 The generating function of the class $\mathcal{C}_{\mathcal{P}}$ with respect to width and volume is given by:

$$
R(t, p)=\frac{t P(p)}{1-t \frac{\partial P}{\partial q}(p)}
$$

In the following section, we examine two possibilities for the Property $\mathcal{P}$ : directed strata are first considered and then vertically convex directed ones.

\section{Enumeration of two classes of polycubes}

\subsection{The s-directed polycubes}

An s-directed polycube can be split into directed strata, as illustrated by Figure 5 . Hence the generic method applies, with an empty property $\mathcal{P}$. We first have to look for the generating function of directed polyominoes with respect to area. It is proved in [DPB82, GBV88, BP93] that this generating function is equal to:

$$
\frac{1}{2}\left(\left(1-\frac{4 q}{1+q}\right)^{-1 / 2}-1\right)
$$

Then, applying Theorem 3.1 to the case of s-directed strata, we get the following proposition:

Proposition 4.1 The generating function of s-directed polycubes with respect to width and volume is given by:

$$
D P(t, p)=-\frac{1}{2} \frac{t\left(1-2 t p-3 p^{2}\right)(1-X(p))}{t p-X(p)\left(1-2 p-3 p^{2}\right)}
$$

where

$$
X(p)=\sqrt{\frac{1-3 p}{1+p}} .
$$

According to Proposition 4.1 and using Maple, the first values of $D P(t, p)$ can easily be obtained. These values are given in Table 1 .

Let us set $D P(t, p)=\sum_{n, m \geq 0} d p_{n, m} t^{n} p^{m}$, where $d p_{n, m}$ is the number of s-directed polycubes of width $n$ and volume $m$. Then, from the values of Table 1 it is possible to extract some marginal values of $D P(t, p)$.

\section{Proposition 4.2}

1. $d p_{n, n}=1$;

2. $d p_{n-1, n}=4 n-6$;

3. $d p_{n-2, n}=8 n^{2}-33 n+32$.

Proof: The proofs of these formulas are based on the following principle. We start from the polycube of height 1 , depth 1 and width $n-i$, with $i \geq 0$, that is a box of volume $n-i$. Next, we build all the polycubes of width $n-i$ and volume $n$ from this box by adding $i$ cells, cutting and gluing them. Thus, we only detail the proof of the value of $d p_{n-2, n}$. Let us consider the box of volume $n-2$ and width $n-2$ 


\begin{tabular}{l|ccccccccc} 
vol/width & 1 & 2 & 3 & 4 & 5 & 6 & 7 & 8 & 9 \\
\hline 1 & 1 & & & & & & & & \\
2 & 2 & 1 & & & & & & & \\
3 & 5 & 6 & 1 & & & & & & \\
4 & 13 & 28 & 10 & 1 & & & & & \\
5 & 35 & 115 & 67 & 14 & 1 & & & & \\
6 & 96 & 441 & 369 & 122 & 18 & 1 & & & \\
7 & 267 & 1617 & 1808 & 839 & 193 & 22 & 1 & & \\
8 & 750 & 5748 & 8188 & 4984 & 1589 & 280 & 26 & 1 & \\
9 & 2123 & 19971 & 35036 & 26765 & 11073 & 2683 & 383 & 30 & 1
\end{tabular}

Table 1: Number of s-directed polycubes with respect to width and volume.

and let us enumerate all the ways to inject two cells to obtain an s-directed polycube. There are two main cases:

- We add two cells to a stratum.

Let us remark that, if we add two cells to a stratum (reduced to a cell), we obtain a directed stratum of volume 3 and that there are 5 different such strata.
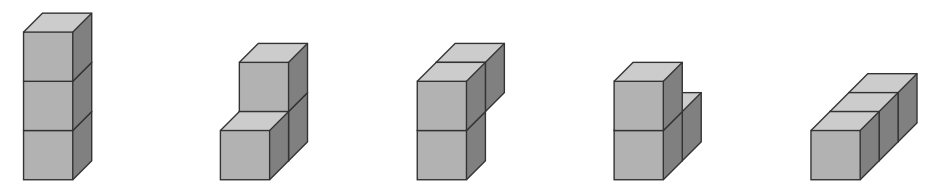

To add two cells to a stratum different from the last one, there are $n-3$ possible strata. Then, we isolate the selected stratum and we substitue it by one of volume 3 ( 5 possibilities, see above). Next, we glue the leftmost part of the initial polycube. There is only one possibility that keeps the direction. Then, we glue the rightmost part of the initial polycube. There are 3 possibilities. Finally, there are 15(n-3) possibile constructions. An example of such a construction is given Figure 6

If we insert the two cells to the last stratum (the rightmost one) of the box, we have 5 possibilities, since there are only 5 strata of volume 3 .

So, if we add two cells to a stratum, we obtain $15 n-40$ different possibilities.

- If we add the two cells to two different strata, we must investigate again two cases:

- No cell is added to the last stratum. Then, there are $\left(\begin{array}{c}n-3 \\ 2\end{array}\right)$ possible choices of strata and $2 \times 2$ choices for each stratum, which gives $(2 \times 2)^{2}\left(\begin{array}{c}n-3 \\ 2\end{array}\right)$ possibilities.

- One cell is added to the last stratum, which lets 2 choices for this one. There are $n-3$ choices for the second stratum, 2 possibilities to add a cell to it and 2 other possibilities to glue this new stratum to the last part of the polycube. So, we have here $8(n-3)$ different choices. 


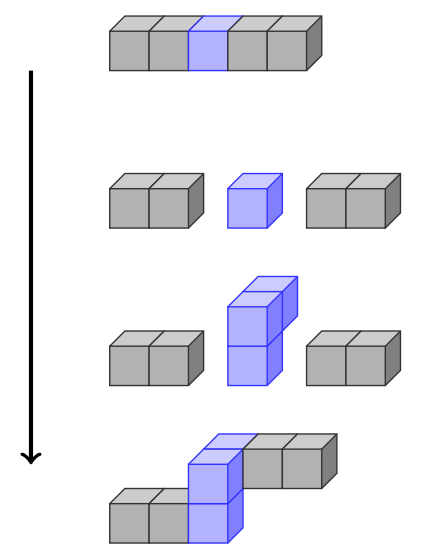

Figure 6: Construction of an s-directed polycube of width 5 and volume 7.

Adding the number of all the possibilities, we obtain that $d p_{n-2, n}=8 n^{2}-33 n+32$.

Let us note that we have not been able to find a formula in the general case. However, it is possible to state the following proposition:

Proposition 4.3 The coefficient $d p_{n-i, n}$ is a polynomial in $\mathbb{Q}[n]$ the highest-degree-term of which is equal to $\frac{4^{i}}{i !} n^{i}$.

Proof: Proposition 4.3 is a particular case of Proposition 6.4 that is proved later. The main interest of the following proof is that it takes into acount the specifics of the 3-dimensional case.

Let us consider the polycube of height 1 , depth 1 and width $n-i$ and let us add to it $i$ cells to $i$ different columns (except for the last one). We have $\left(\begin{array}{c}n-i-1 \\ i\end{array}\right)$ different choices of columns. Then, for each selected column, we have 2 possibilities to inject a cell and keep the property of direction and 2 ways to substitute the initial stratum by the new one in order to obtain a polycube. As we perform this construction for $i$ columns, we finally obtain $4^{i}\left(\begin{array}{c}n-i-1 \\ i\end{array}\right)$ possibilities.

In all the other cases, we only have $\left(\begin{array}{c}n-i-1 \\ i-j\end{array}\right)$ possibilities to choose columns, with $j>1$, leading to a term of degree $i-j$. Since $j>1$, the highest-degree-term in $O\left(n^{i-j}\right)$ is negligible in the presence of terms of $O\left(n^{i}\right)$ order. So, the highest degree term in $n$ is $\frac{4^{i}}{i !} n^{i}$.

Computing a polynomial interpolation of $D P(t, p)$ leads to the following results: 


\section{Corollary 4.1}

$$
\begin{aligned}
d p_{n-3, n} & =\frac{32}{3} n^{3}-84 n^{2}+\frac{622}{3} n-155 \\
d p_{n-4, n} & =\frac{32}{3} n^{4}-136 n^{3}+\frac{3683}{6} n^{2}-\frac{2255}{2} n+660 \\
d p_{n-5, n} & =\frac{128}{15} n^{5}-160 n^{4}+1138 n^{3}-3741 n^{2}+\frac{80452}{15} n-2212 \\
d p_{n-6, n} & =\frac{256}{45} n^{6}-\frac{736}{5} n^{5}+\frac{13604}{9} n^{4}-\frac{46313}{6} n^{3}+\frac{1778963}{90} n^{2}-\frac{317602}{15} n \\
& +2948 \\
d p_{n-7, n} & =\frac{1024}{315} n^{7}-\frac{1664}{15} n^{6}+\frac{69808}{45} n^{5}-\frac{33962}{3} n^{4}+\frac{2022853}{45} n^{3}-\frac{888669}{10} n^{2} \\
& +\frac{3801607}{70} n+38106 \\
d p_{n-8, n} & =\frac{512}{315} n^{8}-\frac{7424}{105} n^{7}+\frac{58096}{45} n^{6}-12732 n^{5}+\frac{25964207}{360} n^{4}-\frac{13520417}{60} n^{3} \\
& +\frac{764855419}{2520} n^{2}+\frac{8527553}{84} n-500380
\end{aligned}
$$

\subsection{The vertically-convex s-directed polycubes}

As for the case of s-directed polycubes, we start by providing an expression for the generating function of vertically-convex s-directed polycubes with respect to volume and width and then, we state some properties of these polycubes.

A vertically-convex s-directed polycube is such that each of its strata is vertically-convex and directed (see Figure 7). Let us remark that such a stratum is in fact a column-convex directed polyomino in which each unit square has been substituted by a unit cube. So, according to the method described in Section 3 . we first have to provide the generating function of column-convex directed polyominoes with respect to area.
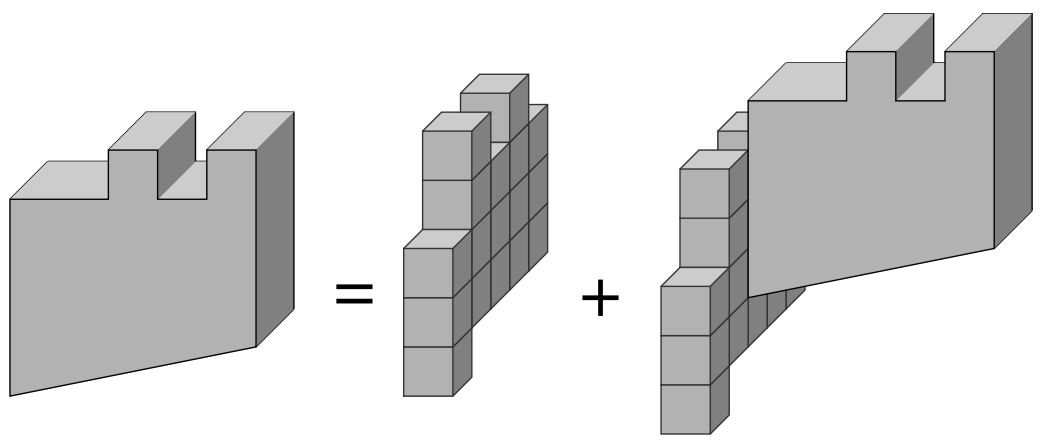

Figure 7: Decomposition of a vertically-convex s-directed polycube. 
In [DD93], Delest and Dulucq show that this generating function is equal to:

$$
\frac{q(q-1)}{1-3 q+q^{2}}
$$

So, using Theorem 3.1, we can deduce the following proposition:

Proposition 4.4 The generating function of vertically-convex s-directed polycubes with respect to width and volume is given by:

$$
V C D(t, p)=\frac{t p(p-1)\left(1-3 p+p^{2}\right)}{1-6 p+11 p^{2}-6 p^{3}+p^{4}-t\left(p-2 p^{2}+2 p^{3}\right)} .
$$

As for the case of s-directed polycubes, we can easily compute the first values of $V C D(t, p)$. They are given in Table 2 .

\begin{tabular}{l|cccccccccc} 
vol/width & 1 & 2 & 3 & 4 & 5 & 6 & 7 & 8 & 9 & 10 \\
\hline 1 & 1 & & & & & & & & \\
2 & 2 & 1 & & & & & & & \\
3 & 5 & 6 & 1 & & & & & & \\
4 & 13 & 28 & 10 & 1 & & & & & \\
5 & 34 & 115 & 67 & 14 & 1 & & & & \\
6 & 89 & 435 & 369 & 122 & 18 & 1 & & & \\
7 & 233 & 1554 & 1797 & 339 & 193 & 22 & 1 & & \\
8 & 610 & 5234 & 8029 & 4968 & 1589 & 280 & 26 & 1 & & \\
9 & 1597 & 17667 & 33640 & 26470 & 11052 & 2683 & 383 & 30 & 1
\end{tabular}

Table 2: Number of vertically-convex s-directed polycubes with respect to width and volume.

Let us set $V C D(t, p)=\sum_{n, m \geq 0} v c d_{n, m} t^{n} p^{m}$, where $v c d_{n, m}$ is the number of vertically-convex sdirected polycubes of width $n$ and volume $m$.

Let us note that all the strata of volume less than or equal to 3 are vertically-convex s-directed. Since each stratum of a polycube of volume $n$ and width $n-i$, with $0 \leq i \leq 2$, has a volume less or equal to 3 , all of these polycubes are both s-directed and vertically-convex s-directed. So, for $0 \leq i \leq 2$, the properties satisfied by $d p_{n-i, n}$ given in Proposition 4.2 are also satisfied by $v c d_{n-i, n}$. Hence the following proposition:

\section{Proposition 4.5}

$$
\begin{aligned}
& \text { 1. } v c d_{n, n}=1 \\
& \text { 2. } v c d_{n-1, n}=4 n-6 \\
& \text { 3. } v c d_{n-2, n}=8 n^{2}-33 n+32 \text {. }
\end{aligned}
$$

Let us note that according to Proposition 4.5 the three first diagonals of Table 1 and Table 2 are identical. Moreover, with a similar proof as for Proposition 4.3 the following proposition can be stated:

Proposition 4.6 The expression $v c d_{n-i, n}$ is a polynomial in $\mathbb{Q}[n]$ the highest-degree-term of which is equal to $\frac{4^{i}}{i !} n^{i}$. 
Finally, a polynomial interpretation of $\operatorname{VCD}(t, p)$ leads to the following corollary:

\section{Corollary 4.2}

$$
\begin{aligned}
v c d_{n-3, n} & =\frac{32}{3} n^{3}-84 n^{2}+\frac{622}{3} n-155 \\
v c d_{n-4, n} & =\frac{32}{3} n^{4}-136 n^{3}+\frac{3683}{6} n^{2}-\frac{2265}{2} n+684 \\
v c d_{n-5, n} & =\frac{128}{15} n^{5}-160 n^{4}+1138 n^{3}-3761 n^{2}+\frac{83512}{15} n-2723 \\
v c d_{n-6, n} & =\frac{256}{45} n^{6}-\frac{736}{5} n^{5}+\frac{13604}{9} n^{4}-\frac{46553}{6} n^{3}+\frac{1839173}{90} n^{2}-\frac{372577}{15} n \\
& +9508 \\
v c d_{n-7, n} & =\frac{1024}{315} n^{7}-\frac{1664}{15} n^{6}+\frac{69808}{45} n^{5}-11374 n^{4}+\frac{2081353}{45} n^{3}-\frac{3016127}{30} n^{2} \\
& +\frac{6993887}{70} n-27090 \\
v c d_{n-8, n} & =\frac{512}{315} n^{8}-\frac{7424}{105} n^{7}+\frac{58096}{45} n^{6}-\frac{38356}{3} n^{5}+\frac{26600687}{360} n^{4}-\frac{14901367}{60} n^{3} \\
& +\frac{1134385699}{2520} n^{2}-\frac{29729225}{84} n+48250
\end{aligned}
$$

\section{The non-directed case}

\subsection{The vertically-convex pseudo-directed polycubes}

\subsection{The method}

The generic method described in Section 3 can be extended to non-directed polycubes as far as their decomposition into strata obey the following the rule: each stratum has to be glued to the previous one using a distinguished cell that must coincide with some cell of the previous stratum. The distinguished cell of a stratum can be, for instance, the lowest cell of the first column. In fact, the distinguished cell plays the same role as the root in the case of directed polycubes. Even if the considered strata are not directed, they are glued according to a similar rule. Let us call pseudo-directed polycube any polycube obeying this rule.

The generic method can be directly applied to pseudo-directed polycubes. This extension can be illustrated by the enumeration of the vertically-convex pseudo-directed polycubes (see Figure 8 ) which are pseudodirected polycubes that can be split into vertically-convex strata. Other families of pseudo-polycubes could be studied, for instance the pseudo-polycubes that can be split into convex strata.

\subsection{The vertically-convex pseudo-directed polycubes}

Since each stratum of a vertically-convex pseudo-directed polycube is vertically-convex, it is in fact a column-convex polyomino in which each unit square has been substituted by a unit cube. In [Kla65], it is proved that the generating function of column-convex polyominoes with respect to area is defined by:

$$
V(q)=\frac{q(1-q)^{3}}{1-5 q+7 q^{2}-4 q^{3}} .
$$




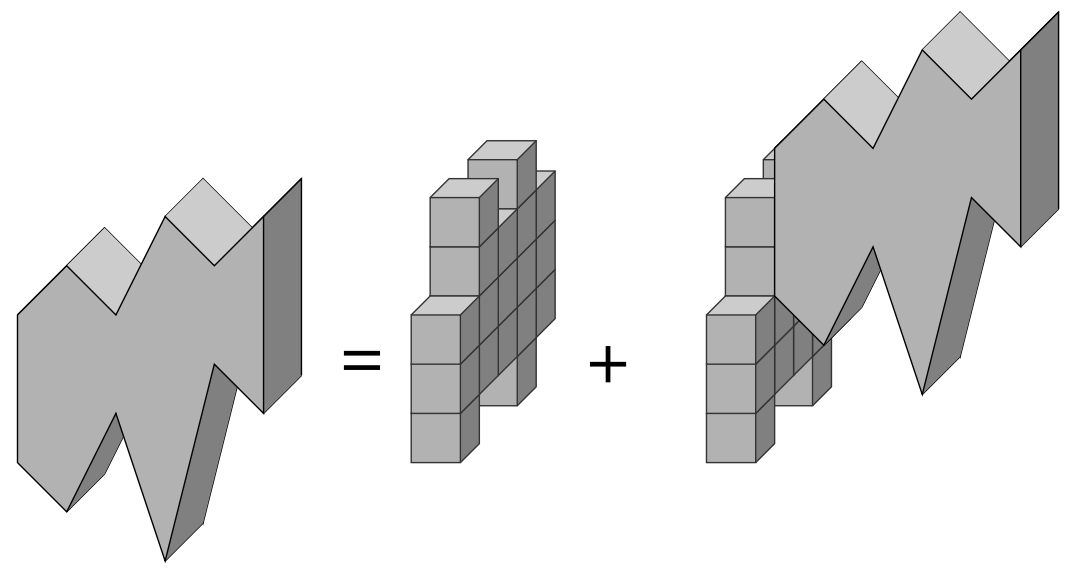

Figure 8: Decomposition of vertically-convex pseudo-directed polycubes.

Thus, the generating function of vertically-convex strata with respect to volume and area of the rightmost face is given by:

$$
\frac{p q(1-p q)^{3}}{1-5 p q+7 p^{2} q^{2}-4 p^{3} q^{3}}
$$

Since the generic method can be applied to pseudo-directed polycubes, according to Theorem 3.1, we obtain:

Proposition 5.1 The generating function $V C(t, p)$ of vertically-convex pseudo-directed polycubes with respect to width and volume, is given by:

$$
V C(t, p)=\frac{t p(1-p)^{3}\left(1-5 p+7 p^{2}-4 p^{3}\right)}{\left(1-5 p+7 p^{2}-4 p^{3}\right)^{2}+t p\left(1-4 p+8 p^{2}-6 p^{3}+4 p^{4}\right)(1-p)^{2}} .
$$

Once again, using Maple, we easily obtain the first values of $V C(t, p)$. These values are listed in the Table 3.

Let us note that the two first diagonals of Table 3 are identical to the two first ones in Table 1 This is because the vertically-convex pseudo-directed polycubes of volume $n$ and width $n$ or $n-1$ are s-directed polycubes. If we set $V C(t, p)=\sum_{n, m \geq 0} v c_{n, m} t^{n} p^{m}$, where $v c_{n, m}$ is the number of vertically-convex pseudo-directed polycubes of width $n$ and volume $m$, we directly obtain, from Proposition 4.2 .

Proposition 5.2

1. $v c_{n, n}=1$,

2. $v c_{n-1, n}=4 n-6$.

Moreover, according to a proof similar as the one of Proposition 4.3 or of Proposition 4.6, it can be shown that:

Proposition 5.3 The expression $v c_{n-i, n}$ is a polynomial in $\mathbb{Q}[n]$ the highest-degree-term of which is equal to $\frac{4^{i}}{i !} n^{i}$. 


\begin{tabular}{l|ccccccccc} 
vol/width & 1 & 2 & 3 & 4 & 5 & 6 & 7 & 8 & 9 \\
\hline 1 & 1 & & & & & & & & \\
2 & 2 & 1 & & & & & & & \\
3 & 6 & 6 & 1 & & & & & & \\
4 & 19 & 32 & 10 & 1 & & & & & \\
5 & 61 & 155 & 74 & 14 & 1 & & & & \\
6 & 196 & 702 & 467 & 132 & 18 & 1 & & & \\
7 & 629 & 3024 & 2659 & 1019 & 206 & 22 & 1 & & \\
8 & 2017 & 12540 & 14060 & 6924 & 1875 & 296 & 26 & 1 & \\
9 & 6466 & 50490 & 70271 & 42952 & 14745 & 3099 & 402 & 30 & 1
\end{tabular}

Table 3: Number of vertically-convex pseudo-directed polycubes with respect to width and volume.

According to Proposition 5.1 and Proposition 5.3 a polynomial interpretation of $V C(t, p)$ leads to the following corollary:

\section{Corollary 5.1}

$$
\begin{gathered}
v c_{n-2, n}=8 n^{2}-30 n+24 \\
v c_{n-3, n}=\frac{32}{3} n^{3}-72 n^{2}+\frac{400}{3} n-45 \\
v c_{n-4, n}=\frac{32}{3} n^{4}-112 n^{3}+\frac{1078}{3} n^{2}-277 n-204 \\
v c_{n-5, n}=\frac{128}{15} n^{5}-128 n^{4}+\frac{1880}{3} n^{3}-864 n^{2}-\frac{5756}{5} n+2380 \\
v c_{n-6, n}=\frac{256}{45} n^{6}-\frac{576}{5} n^{5}+\frac{7184}{9} n^{4}-1756 n^{3}-\frac{126986}{45} n^{2}+\frac{73541}{5} n \\
-11404 \\
v c_{n-7, n}=\frac{1024}{315} n^{7}-\frac{256}{3} n^{6}+\frac{35776}{45} n^{5}-\frac{7760}{3} n^{4}-\frac{180632}{45} n^{3}+42682 n^{2} \\
-\frac{2623083}{35} n-21033 \\
v c_{n-8, n}=\frac{512}{315} n^{8}-\frac{5362}{105} n^{7}+\frac{5824}{9} n^{6}-2944 n^{5}-\frac{158866}{45} n^{4}+\frac{1176386}{15} n^{3} \\
-\frac{9883519}{42} n^{2}+\frac{6748955}{42} n+131860
\end{gathered}
$$

\section{The higher dimensional case}

We first generalize some parameters and definitions from 3-dimensional polycubes to $d$-dimensional ones, with $d \geq 4$. Then we extend the 3 -dimensional enumerative method of Section 3 to higher dimensions. Finally, we apply the extended method to a new family of polycubes that we call the rs-directed d-polycubes. 


\subsection{The extension to $d$-dimensional polycubes}

Let us now consider $\mathbb{N}^{d}$, with $d \geq 3$. An elementary cell is a unitary $d$-cube. A $d$-polycube is then a $d$-face connected finite set of elementary cells, defined up to a translation. The volume of a $d$-polycube is the number of its elementary cells.

Let $\left(0, \overrightarrow{i_{1}}, \ldots, \overrightarrow{i_{d}}\right)$ be an orthonormal coordinate system. The width (resp. height) of a $d$-polycube is the difference between its greatest index and its smallest index according to $\overrightarrow{i_{1}}$ (resp. $\overrightarrow{i_{2}}$ ). A $d$-polycube is said to be vertically-convex if its intersection with any hyperplane $\left(\overrightarrow{i_{2}}, \ldots, \overrightarrow{i_{d}}\right)$ is a vertically-convex $(d-1)$-polycube. It is said horizontally-convex if its intersection with any hyperplane $\left(\overrightarrow{i_{1}}, \overrightarrow{i_{3}}, \overrightarrow{i_{4}}, \ldots, \overrightarrow{i_{d}}\right)$ is a horizontally-convex $(d-1)$-polycube. Other convexities can be defined, but they are not useful here. The notion of step is extended as follows. A step in direction $j$ is a positive move of one unit along the axis $\overrightarrow{i_{j}}$. Then a directed $d$-polycube is such that each cell can be reached from a distinguished one, the root, by a path only made of steps in direction $j$, with $1 \leq j \leq d$. A $d$-stratum of a $d$-polycube is a $d$-polycube of width 1 . We will say stratum instead of $d$-stratum if there is no ambiguity. For $d \geq 3$, the rightmost face of the $i$ th stratum is the $d$-face of this stratum on which is glued the $(i+1)$ th stratum and its surface is the number of its cells. The $(i+1)$ th stratum is called the rightmost stratum of the $i$ th one.

Due to these definitions, the generic method introduced in Section 4.1 can be extented for dimensions higher than 3 . Let $\mathcal{C}_{\mathcal{P}}$ be the class of directed $d$-polycubes the strata of which satisfy property $\mathcal{P}$ and $S(t, p)$ be the generating function for the class $\mathcal{C}_{\mathcal{P}}$ with respect to width and volume. Then, assuming that $P(q)$ is an expression for the generating function of strata in dimension $d-1$ with respect to volume, Theorem 3.1 can be applied, leading to the following proposition:

Proposition 6.1 The generating function $S(t, p)$ is given by

$$
S(t, p)=\frac{t P(p)}{1-t \frac{\partial P}{\partial q}(p)}
$$

\subsection{Enumeration of rs-directed d-polycubes}

Let us consider the family of rs-directed d-polycubes recursively defined as follows. A rs-directed $d$ polycube is:

- either an s-directed polycube if $d=3$,

- or a directed $d$-polycube that can be split into strata that are rs-directed $(d-1)$-polycubes, if $d \geq 4$.

For $d \geq 4$, let us denote by $C^{d}(t, p)$ the generating function for rs-directed $d$-polycubes with respect to width and volume.

We first investigate the case where $d=4$, in order to illustrate how the extension works. From the generating function of s-directed 3-polycubes computed in Section 4.1 and from Proposition 6.1, we can state the following proposition.

Proposition 6.2 The generating function $C^{4}(t, p)$ is given by:

$\frac{(1+p)^{2} X(p)\left(-X(p)+2 X(p) p+3 X(p) p^{2}+p\right)(X(p)-1)(-1+3 p) t}{2(1+p)\left(\left(-19 p^{2}+27 p^{4}+8 p-1\right) X(p)+18 p^{3}-12 p^{2}+2 p\right)+t p\left(\left(3 p^{3}+3 p^{2}+p+1\right) X(p)+\left(27 p^{3}-9 p+9 p^{2}+1\right)\right)}$

where 


$$
X(p)=\sqrt{\frac{1-3 p}{1+p}} .
$$

The first values of $C^{4}(t, p)$ can be easily computed. Setting $C^{4}(t, p)=\sum_{n, m>0} c_{n, m}^{4} t^{n} p^{m}$, where $c_{n, m}^{4}$ is the number of rs-directed 4-polycubes of width $n$ and volume $m$, it is possible, for small values of $i$, to compute $c_{n-i, n}^{4}$ as a polynomial of order $i$. The following proposition holds:

Proposition 6.3 The coefficient $c_{n-i, n}^{4}$ is a polynomial in $\mathbb{Q}[n]$ whose highest-degree-term is equal to $\frac{6^{i}}{i !} n^{i}$

Proof: The proof is based on the same principle as in Proposition 4.3. It is detailed here since it is the main step toward a generalization. Let us consider the 4-polycube $P$ of width and volume equal to $n-i$. Note that each stratum of $P$ is reduced to one cell. Let us inject a cell into $i$ different strata of $P$ (different from the last one): there are $\left(\begin{array}{c}n-i-1 \\ i\end{array}\right)$ different possibilities to choose these strata. Moreover, injecting a cell into a stratum, that is a rs-directed 4-polycube, is equivalent to injecting a cell into a rs-directed 3-polycube. So, we have to inject a cell into a rs-directed 3-polycube reduced to one cell, the root. Since there are 3 possible directions, there are 3 ways to glue a new cell to the root (see Figure 9).

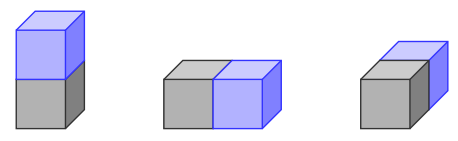

Figure 9: The three ways to inject a cell on a stratum of volume 1.

The end of the proof is similar to the one of Proposition 4.3 , leading to the result that the highest degree term in $n$ is $\frac{6^{i}}{i !} n^{i}$.

A polynomial interpretation of $C^{4}(t, p)$ leads to the following corollary:

\section{Corollary 6.1}

$$
\begin{aligned}
& c_{n, n}^{4}= 1 \\
& c_{n-1, n}^{4}=6 n-9 \\
& c_{n-2, n}^{4}=18 n^{2}-72 n+66 \\
& c_{n-3, n}^{4}=36 n^{3}-270 n^{2}+622 n-420 \\
& c_{n-4, n}^{4}=54 n^{4}-648 n^{3}+2706 n^{2}-4504 n+2352 \\
& c_{n-5, n}^{4}=\frac{324}{5} n^{5}-1134 n^{4}+7416 n^{3}-22074 n^{2}+\frac{142566}{5} n-11445 \\
& c_{n-6, n}^{4}=\frac{16384}{45} n^{6}-\frac{41984}{5} n^{5}+\frac{678464}{9} n^{4}-\frac{991868}{3} n^{3}+\frac{32619376}{45} n^{2}-\frac{10746878}{15} n+226768
\end{aligned}
$$

The case where $d=4$ allowed us to show how the extension works. We also investigated the case of higher dimensions where similar results could be reported. These investigations lead us to the following proposition. 
Proposition 6.4 Let $d \geq 3$ and $C^{d}(t, p)=\sum_{n, m \geq 0} c_{n, m}^{d} t^{n} p^{m}$. Then, the expresssion $c_{n-i, n}^{d}$ is a polynomial in $\mathbb{Q}[n]$ whose highest-degree-term is equal to $\frac{2^{i}(d-1)^{i}}{i !} n^{i}$.

Proof: The proof is essentially based on the fact that adding a cell to a stratum of a rs-directed $d$-polycube is equivalent to inject a cell into an rs-directed $(d-1)$-polycube. As a consequence, there is a unique possibility to add a cell to a $d$-stratum of volume 1 with respect to each of the $d-1$ directions. Then, the rightmost stratum initially glued to the modified stratum can be glued again in two different ways in order to satisfy the property of direction of rs-directed d-polycubes. Note that the last stratum is not concerned by this construction since it has no rightmost stratum. So, there are $2(d-1)$ possible constructions for the $i$ selected strata, which leads to $(2(d-1))^{i}\left(\begin{array}{c}n-i-1 \\ i\end{array}\right)$ possibilities to realize this operation. If the last stratum belongs to the set of selected strata, there are only $2^{i} \times(d-1)^{i-1}\left(\begin{array}{c}n-i-2 \\ i\end{array}\right)$ possibilities. In all the other cases, there are $\left(\begin{array}{c}n-i-1 \\ i-j\end{array}\right)$ or $\left(\begin{array}{c}n-i-2 \\ i-j\end{array}\right)$ (and $j>1$ ) possibilities to choose strata, leading to a term of degree $i-j$. Since $j>1$, the highest-degree-term in $O\left(n^{i-j}\right)$ is negligible with respect to $n^{i}$. Finally, the highest-degree-term of $c_{n-i, n}^{d}$ is equal to $\frac{2^{i}(d-1)^{i}}{i !} n^{i}$.

\section{Conclusion}

A lot of results are known about the enumeration of polyominoes, at least for specific classes of polyominoes. But the transition from dimension 2 to dimension 3 (or to a higher dimension) is known to be a hard combinatorial problem and very few results are known about polycubes. This paper is a significant advance on this topic. It introduces a generic method that allows us to enumerate various classes of directed $d$-polycubes, for $d \leq 3$, as far as their strata satisfy a given property $\mathcal{P}$. This method can be applied both to directed polycubes, such as the s-directed 3-polycubes or the vertically-convex s-directed 3-polycubes, and to non-directed polycubes, such as the vertically-convex pseudo-directed 3-polycubes. Moreover, it can be applied in any dimension: an example is the class of s-directed $d$-polycubes, with $d \geq 3$. For each of the above-mentioned classes of $d$-polycubes, the generating function with respect to width and volume is exhibited whereas the number of $d$-polycubes of volume $n$ and width $n-i$ is obtained by a simple method and shown to be a polynomial of degree $i$. In the future, we intend to follow this track and to investigate other ones in order to extend the enumeration to other classes of $d$-polycubes.

\section{Acknowledgements}

We would like to thank anonymous referees of an earlier conference version for helpful and constructive remarks and referee of this version for a careful proofreading. 


\section{References}

[AB09] G. Aleksandrowicz and G. Barequet. Counting $d$-dimensional polycubes and nonrectangular planar polyominoes. Int. J. Comput. Geometry Appl., 19(3):215-229, 2009.

[BH57] S.R. Broadbent and J.M. Hammersley. Percolation processes i. crystals and mazes. In Proceedings of the Cambridge Philosophical Society, volume 53, pages 629-641, 1957.

[BM96] M. Bousquet-Mélou. A method for the enumeration of various classes of column-convex polygons. Discrete Mathematics, 154(1-3):1-25, 1996.

[BP93] J. Bétréma and J. G. Penaud. Animaux et arbres guingois. Theor. Comput. Sci., 117(1\&2):6789, 1993.

[Bre99] D.M. Bressoud. Proofs and Confirmations: The Story of the Alternating Sign Matrix Conjecture. Spectrum Series. Cambridge University Press, 1999.

[CDJ09] J.-M. Champarnaud, J.P. Dubernard, and H. Jeanne. An efficient algorithm to test whether a binary and prolongeable regular language is geometrical. Int. J. Found. Comput. Sci., 20(4):763$774,2009$.

[CDJ10] J.-M. Champarnaud, J.P. Dubernard, and H. Jeanne. Enumeration of specific classes of polycubes. In Lattice Path Combinatorics and Applications, pages 79-87, Siena, Italy, 2010.

[CLP98] H. Cohn, M. Larsen, and J. Propp. The shape of a typical boxed plane partition. New York J. Math., 4:137-166, 1998.

[DD93] M.P. Delest and S. Dulucq. Enumeration of directed column-convex animals with given perimeters and area. In Croatica Chimica Acta, volume 66, pages 59-80, 1993.

[DF93] M.P. Delest and J.M. Fédou. Enumeration of skew Ferrers diagrams. Discrete Mathematics, 112(1-3):65-79, 1993.

[DPB82] D. Dhar, M. K. Phani, and M. Barma. Enumeration of directed site animal on two-dimensional lattices. J. Phys. A, 15:279-284, 1982.

[DV84] M.P. Delest and X.G. Viennot. Algebraic languages and polyominoes enumeration. Theor. Comput. Sci., 34:169-206, 1984.

[Fer04] S. Feretić. A q-enumeration of convex polyominoes by festoon approach. Theoret. Comput. Sci., 319(1-3):333-356, 2004.

[GBV88] D. Gouyou-Beauchamps and G. Viennot. Equivalence of the two-dimensional directed animal problem to a one-dimensional path problem. Advances in Applied Mathematics, 9(3):334 $357,1988$.

[Jea10] H. Jeanne. Langages géométriques et polycubes. PhD thesis, Université de Rouen, France, 2010. 
[Kla65] D. Klarner. Some results concerning polyominoes. In Fibonnacci Quart., volume 3, pages 9-20, 1965.

[LG08] G. Largeteau and D. Geniet. Quantification du taux d'invalidité d'applications temps-réel à contraintes strictes. Technique et Science Informatiques, 27(5):589-625, 2008.

[Lun71] W.F. Lunnon. Counting polyominoes. In Computers in Number Theory, pages 347-372. A.O.L. Atkin and B.J. Birch, Academic Press, London, 1971.

[Sta01] R.P. Stanley. Enumerative combinatorics. 2, chapter 7. Cambridge Studies in Advanced Math. Wadsworth \& Brooks/Cole Advanced Books \& Software, 2001.

[Tem56] H. N. V. Temperley. Combinatorial problems suggested by the statistical mechanics of domains and rubber-like molecules. Phys. Rev., 103:1-16, 1956. 